\title{
Effect of Post-Harvest Application of Biocides on Vase Life of Cut Gerbera (Gerbera jamesonii Bolus ex. Hook) cv. Alppraz
}

\author{
P. Hema ${ }^{1}$, V. Vijaya Bhaskar ${ }^{2}$, A.V.D. Dorajeerao ${ }^{1}$ and D.R.S. Suneetha ${ }^{2 *}$ \\ ${ }^{1}$ Department of Floriculture and Landscape Architecture, ${ }^{2}$ Department of Plant Biochemistry, \\ College of Horticulture, Dr. Y. S. R. Horticultural University, Venkataramannagudem - 534 \\ 101, West Godavari District, Andhra Pradesh, India \\ *Corresponding author
}

\section{A B S T R A C T}

The present investigation was carried out at College of Horticulture, Dr. Y.S.R. Horticultural University, Venkataramannagudem, West Godavari district of Andhra Pradesh during the year 2013-2014. The experiment was conducted with two different types of biocides viz., sodium hypochlorite and calcium hypochlorite each at three

\section{Keywords}

Electrolytes leakage, Gerbera, Total sugars, Vase life, Water uptake, Water balance

Article Info

Accepted:

20 February 2018

Available Online:

10 March 2018 concentrations along with control (distilled water only), a total of seven treatments with three replications in a completely randomised design with factorial concept. Aim of the experiment was to find out influence of these chemicals to check the proliferation of microbes in the vase solution in order to improve the water relations in the floral tissue. Flowers held in the vase solution contained sodium hypochlorite $20 \mathrm{ppm}$ recorded significantly longest vase life (10.570 days) which might be attributed to an improvement in the water relations of the floral tissue through an increase in the total water uptake (8.089 g/flower spike), reduced transpiration loss of water (8.405 g/flower spike), improved water balance (3.753 g/flower spike) and fresh weight change of flowers ( $100.463 \%$ of initial of flower weight). An increase noticed with regard to total sugars content $(3.700 \mathrm{mg} / \mathrm{g}$ fresh weight) and a reduction observed in the electrolytes leakage $(27.738 \%)$ of flower petals by application of sodium hypochlorite $20 \mathrm{ppm}$ also might have contributed to improved vase life. The same treatment recorded significantly lower values with regard to scape bending curvature (10.017 degrees) of the flower and optical density (0.034) of vase solution when compared with all other treatments, thus contributed to improved quality and vase life of cut gerbera flowers.

\section{Introduction}

Gerbera (Gerbera jamesonii Bolus ex. Hook), commonly known as Transvaal daisy, Barberton daisy or African daisy, belongs to the family Asteraceae. It is one of the most popular commercial cut flowers grown throughout the world under a wide range of climatic conditions for its attractive flowers. It is the fourth most important cut flower in the world trade after rose, carnation and chrysanthemum. Gerbera is believed to be native to South Africa and Asiatic region. Gerbera is now one of the popular cut flower 
crops grown worldwide with increasing commercial significance in floricultural trade due to wide range of flower colours except blue. The flowers may be single or double and are available in various self-coloured cultivars as well as in bi-coloured. The attractive blooms of gerbera are suitable for any type of floral arrangement like preparation of bouquets, floral ornaments and in making dry flower crafts. Beauty of cut flower lies with the freshness of flower for a reasonably prolonged period of time without loosing its aesthetic value. Postharvest longevity in cut flowers is not length of lasting quality in itself but the satisfaction of the consumer (Buys, 1978). Vase life is often used as an indicator of postharvest longevity in cut flowers. As cut flowers are vulnerable to heavy postharvest losses, they lose their vase life in a very short period of time. This cause's great loss in cut flower market, hence there is a great need to compensate the loss to develop suitable postharvest technology specific to each cut flower to reach higher market price.

The extension of cut flower vase life with improved postharvest handling and maintenance has now become commercial and economically important based on scientific principles. Once the flower is separated from its mother plant, the continuity of water to the flower is disturbed, as water relations play an important role in the postharvest physiology of cut flowers (Halevy and Mayak, 1981). Accomplishing extension of vase life depends on postharvest handling and use of a suitable preservative solution ensuring ample supply of water and metabolites in addition to reserved food material in the stems and petals (Halevy and Mayak, 1979).

The postharvest longevity of cut flowers having economic value can often be improved by use of different chemicals and sugars in vase solution (Halevy et al., 1978; Murali, 1990; Emongor, 2004; Prashanth, 2006). An effective flower food i.e., a preservative solution should contain three basic components to extend the life of cut flowers. A sugar to provide energy to the flowers, a biocide to kill the microbes and an acidifier to lower the $\mathrm{pH}$ of solution which increases and maintains the uptake of water and nutrients by flower spike (Coake, 1997).

Improper postharvest handling and short life of cut flower (Wernett et al., 1996) are the major problems associated with gerbera cut flowers. Vase life of cut gerbera flowers is often limited by bending of flower stalk called as scape bending (Wilberg, 1973 and Fischer et al., 1982). Pre-mature senescence is another problem associated with shortened vase life of gerberas. Lack of proper postharvest technical knowledge about floriculture in general and gerbera in particular is a major constraint in gerbera cultivation in India. Keeping all these constraints in view, the present investigation was designed to assess the effect of different biocides on the longevity of cut gerbera flowers.

\section{Materials and Methods}

The present experiment was carried out at the Post Graduate Research Laboratory of Floriculture and Landscape Architecture at College of Horticulture, Venkataramannagudem, West Godavari district of Andhra Pradesh during the year 2013-2014. Fresh flowers of gerbera (Gerbera jamesonii) cultivar 'Alppraz' obtained from a commercial greenhouse located in close proximity to the experimental site were used for the present experimentation. Cut gerbera flowers used in the present investigations were grown under naturally ventilated polyhouse conditions with all recommended fertigation and pest management practices. Flowers were harvested from one year old mother plant at the commercial stage (ray florets $3 / 4^{\text {th }}$ opened) in the morning hours between 6.30 am and 7.30 am by pulling the scape of length ranging $50-60 \mathrm{~cm}$ from the crown. Immediately after 
harvest, $5 \mathrm{~cm}$ of basal woody portion was cut under distilled water and brought to the laboratory by immersing base of the flowers in distilled water. The flowers were pre-cooled at $4 \pm 2{ }^{0} \mathrm{C}$ for about $4 \mathrm{~h}$ and then immediately sorted out to uniform length and quality of capitulum in order to maintain uniformity within the replications. Flower scapes were trimmed under water to $40 \mathrm{~cm}$ length as Lemper (1981) suggested that cleaning the stems and re-cutting the base before placing them in the solution were found essential. The gerbera cultivar Alppraz is a stem less perennial herb. The leaves are elongated, lobed, texture of the leaf is coarse. Scapes bear red flowers with ray, trans and disc florets. Disc florets are smaller, numerous, trans florets are short with pappus of rough bristles in many rows. Capitulum was of 7.5-12.5 cm diameter. Scape was solitary, 50-60 cm long with woody base.

The experiment was conducted with seven treatments in a completely randomized design with three replications. The biocides used in the present experiment were sodium hypochlorite and calcium hypochlorite each with two concentrations. The treatment details were: $\mathrm{T}_{1}$ : Sodium Hypochlorite $20 \mathrm{ppm}$ ( $\mathrm{SH}$ 20); $\mathrm{T}_{2}$ : Sodium Hypochlorite $40 \mathrm{ppm}$ ( $\mathrm{SH}$ 40); $\mathrm{T}_{3}$ : Sodium Hypochlorite $60 \mathrm{ppm}$ ( $\mathrm{SH}$ 60); $\mathrm{T}_{4}$ : Calcium Hypochlorite $20 \mathrm{ppm}(\mathrm{CH}$ 20); $\mathrm{T}_{5}$ : Calcium Hypochlorite $40 \mathrm{ppm}(\mathrm{CH}$ 40); $\mathrm{T}_{6}$ : Calcium Hypochlorite $60 \mathrm{ppm}(\mathrm{CH}$ 60); $\mathrm{T}_{7}$ : Control (Distilled water). The available active ingredient in sodium hypochlorite was only 4\%. To get 1000 ppm of sodium hypochlorite, $25 \mathrm{ml}$ of $4 \%$ sodium hypochlorite was dissolved in $1000 \mathrm{ml}$ of distilled water and from the stock solution, the necessary dilutions were made to obtain required concentrations. The available active ingredient in calcium hypochlorite was only $30 \%$. To get $1000 \mathrm{ppm}$ of calcium hypochlorite, $3.33 \mathrm{~g}$ of $30 \%$ calcium hypochlorite was dissolved in $1000 \mathrm{ml}$ of distilled water and from the stock solution, the necessary dilutions were made to obtain required concentrations. The flowers were continuously held in the treatment solution (Holding solution) till the end of the vase life period. Vase life is defined as days from the time of immersion in the test solution to the loss of ornamental value. Same treatments were repeated for destructive samples, used for physiological and biochemical studies. In each glass bottle $350 \mathrm{ml}$ of aqueous test solution / holding solution of different treatments was filled and their weight was recorded. Then five flowers were placed in each bottle and considered as one replication and their initial fresh weights were recorded. The mouth of the bottles was sealed with aluminium foil which effectively prevents the evaporational loss of aqueous test solutions. The weight of each container and the test solution / distilled water with and without flower scapes was recorded for every two days. While recording weights, re-cutting of the floral stems (about $0.5 \mathrm{~cm}$ ) was done under distilled water. Vase life and other visual observations of the flowers were recorded daily. Water uptake, transpiration loss of water, water balance, fresh weight change, electrolytes leakage and vase life were measured as described by Bhaskar (2001). Optical density of vase solution was measured at every alternate day by using spectrophotometer (Spectrophotometer 166) at $480 \mathrm{~nm}$. Scape bending curvature was measured as per the procedure explained by van Doorn et al., (1994). Total sugars content in the flower petals was measured as per the procedure explained by Dubois et al., (1956). The data arrived was analysed statistically by following the standard statistical methods outlined by Panse and Sukhatme (1985).

\section{Results and Discussion}

Application of biocides in the holding solution recorded significant variations in the water uptake during vase life period of cut gerbera (Table 1). Among the biocide concentrations, 
sodium hypochlorite 20 ppm recorded significantly highest water uptake (8.089 g/flower) followed by sodium hypochlorite 40 ppm. Control recorded significantly lowest water uptake (4.431 g/flower). Significant differences were observed in the water uptake during different days of vase life period. Significantly highest water uptake was observed on day 2 (9.407 g/flower), whereas, significantly lowest water uptake was observed on day 10 (3.107 g/flower). Interaction effect of water uptake between treatments and days was found significant. Sodium hypochlorite $20 \mathrm{ppm}$ recorded significantly highest water uptake during the entire period of evaluation. Control recorded significantly lowest water uptake on all the days of experimentation. Increased water uptake noticed with lower concentration of sodium hypochlorite was considered to be very effective even at a low concentration in reducing the microbial growth thereby avoiding stem blockage and maintained continuity of water to the floral tissue. Marousky (1969) expressed similar kind of opinion while working with cut roses. Anju and Santhosh (2004) reported similar kind of observation while working with cut gladiolus spikes.

Significant differences were noticed in the transpiration loss of water of cut gerbera by employing different biocides at different concentrations in the holding solution (Table 1). Sodium hypochlorite $20 \mathrm{ppm}$ concentration recorded significantly highest transpiration loss of water (8.405 g/flower) followed by sodium hypochlorite $40 \mathrm{ppm}$ (7.971 g/flower). Control recorded significantly lowest transpiration loss of water (5.379 g/flower). Significant differences were observed in the transpiration loss of water during different days of vase life period. A continuous and significant decrease was noticed in the transpiration loss of water at each successive interval of observation recorded during vase life period. Significantly highest transpiration loss of water was noticed on day 2 (9.235 g/flower), whereas, significantly lowest transpiration loss of water was observed on day 10 (4.369 g/flower). Interaction effect between days and treatments on transpiration loss of water was also found significant. Sodium hypochlorite $20 \mathrm{ppm}$ recorded significantly highest transpiration loss of water on all the days of observation recorded followed by sodium hypochlorite $40 \mathrm{ppm}$. Control recorded significantly lowest transpiration loss of water during the period of vase life evaluation. Increased water uptake might have led to an increase in the transpiration loss of water mainly to avoid the temporary stress (Halevy et al., 1978) in the plant tissue which led to an increase in the membrane viscosity (Faragher, 1986) of the cell. Significantly lowest transpiration loss of water observed in control was mainly due to lower water uptake thereby quantity of water retained in the floral tissue was found meagre which led to wilting of cut flowers in advance. The result obtained by Balakrishna et al., (1989) in cut tuberose spikes was found in tune with the present result.

Significant differences recorded in the water balance of cut gerbera flowers held in different biocide solutions (Table 2). Cut gerbera flowers held in sodium hypochlorite at $20 \mathrm{ppm}$ concentration recorded significantly highest water balance (3.753 g/flower) followed by sodium hypochlorite at $40 \mathrm{ppm}$ concentration (3.508 g/flower). However, cut gerbera flowers held in calcium hypochlorite at $60 \mathrm{ppm}$ concentration recorded significantly lowest water balance (3.045 g/flower). Significant differences were observed in the water balance of cut gerbera flowers during different days of vase life period. Significantly highest water balance was observed on day 2 (4.170 g/flower), whereas, on day 10 significantly lowest water balance (2.733 g/flower) was observed. A significant decrease was noticed in the water balance from the beginning of the experiment to the end of the 
experiment. Interaction effect between days and treatments on waster balance was also found significant. Sodium hypochlorite 20 ppm recorded significantly highest water balance on day 4, 8 and 10 (4.710, 3.243 and $3.237 \mathrm{~g} /$ flower respectively), whereas, sodium hypochlorite $60 \mathrm{ppm}$ recorded significantly highest water balance on day 2 (4.610 $\mathrm{g} /$ flower) and was found at par with sodium hypochlorite $20 \mathrm{ppm}$ (3.043 g/flower) as well as with calcium hypochlorite $40 \mathrm{ppm}$ concentration (3.070 g/flower). Significantly lowest water balance was noticed with control on day 4,8 and 10 (3.850, 2.367 and 2.343 g/flower), whereas, on day 2 calcium hypochlorite $60 \mathrm{ppm}$ recorded significantly lowest water balance (3.427 g/flower), on day 6 sodium hypochlorite $60 \mathrm{ppm}$ recorded significantly lowest water balance $(2.480$ $\mathrm{g} /$ flower). Cut gerbera flowers held in $\mathrm{NaOCl}_{2}$ $20 \mathrm{ppm}$ developed a negative water balance much later than those held in other treatments almost during the entire period of evaluation. Sodium hypochlorite $20 \mathrm{ppm}$ exerted a beneficial effect on water balance of cut gerberas, which might be attributed to its effect on stomatal closure (Stoddard and Miller, 1962 and Bhaskar, 2001) thus reducing the transpiration loss of water.

Significant variation was recorded in the fresh weight change of cut gerbera flowers evaluated for their vase life using biocides in the holding solution (Table 2). Sodium hypochlorite $20 \mathrm{ppm}$ concentration recorded significantly highest fresh weight change $(100.463 \%)$ followed by calcium hypochlorite 20 ppm (98.127\%). Control recorded significantly lowest fresh weight change $(89.238 \%)$. Significant differences were observed in the fresh weight change during different days of vase life of cut gerbera. Significantly highest fresh weight change was observed on day 2 (106.851\%), whereas, significantly lowest fresh weight change was noticed on day $10(84.915 \%)$. The interaction effect between days and treatments on fresh weight change was also found significant. Sodium hypochlorite $20 \mathrm{ppm}$ concentration recorded significantly highest fresh weight change on almost all the days of evaluation, whereas, control recorded significantly lowest fresh weight change on almost all the days of observation. An increase in fresh weight could be attributed to increased water uptake and decreased transpiration loss of water in the floral tissue, thus improved water balance in the floral tissue. Similar kind of observation was also reported by several research workers including Larsen and Frolich (1969) in cut carnations, Marousky (1969) and Bhattacharjee (1998) in cut roses, De Jong (1978) and Prashanth (2006) in cut gerberas.

Significant differences were observed in the electrolytes leakage of cut gerbera flowers held in different biocide solutions (Table 3). Significantly lowest electrolytes leakage was noticed with sodium hypochlorite $20 \mathrm{ppm}$ concentration $(27.738 \%)$ followed by sodium hypochlorite $40 \mathrm{ppm}$ concentration $(28.869 \%)$. Control recorded significantly highest electrolytes leakage $(32.671 \%)$. Significant differences were observed in the electrolytes leakage during different days of vase life period of cut gerbera flowers. Significantly lowest electrolytes leakage was noticed on day $2(21.357 \%)$, whereas, significantly highest electrolytes leakage was observed on day 10 $(37.907 \%)$.

Interaction effect between days and treatments on electrolytes leakage was also found significant. On day 2, no significant differences were observed in the electrolytes leakage among the treatment combinations, whereas, during the remaining period of evaluation, sodium hypochlorite $20 \mathrm{ppm}$ concentration recorded significantly lowest electrolytes leakage followed by sodium hypochlorite $40 \mathrm{ppm}$. 
Table.1 Effect of postharvest application of biocides on water uptake and transpiration loss of water during Vase life period of cut gerbera

\begin{tabular}{|c|c|c|c|c|c|c|c|c|c|c|c|c|}
\hline \multirow{3}{*}{ Treatments $(\mathbf{T})$} & \multicolumn{12}{|c|}{ Days (D) } \\
\hline & \multicolumn{6}{|c|}{ Water uptake (g) } & \multicolumn{6}{|c|}{ Transpiration loss of water $(\mathrm{g})$} \\
\hline & $2^{\text {nd }}$ & $4^{\text {th }}$ & $6^{\text {th }}$ & $\mathbf{8}^{\text {th }}$ & $10^{\text {th }}$ & Mean & $2^{\text {nd }}$ & $4^{\text {th }}$ & $6^{\text {th }}$ & $8^{\text {th }}$ & $10^{\text {th }}$ & Mean \\
\hline Sodium hypochlortie 20 ppm & 11.070 & 9.923 & 7.833 & 7.573 & 4.047 & 8.089 & 10.533 & 9.213 & 9.123 & 8.340 & 4.813 & 8.405 \\
\hline Sodium hypochlortie $40 \mathrm{ppm}$ & 10.613 & 9.173 & 7.277 & 6.700 & 3.653 & 7.483 & 10.183 & 9.247 & 8.100 & 7.660 & 4.663 & $\mathbf{7 . 9 7 1}$ \\
\hline Sodium hypochlortie $60 \mathrm{ppm}$ & 9.760 & 8.160 & 6.380 & 5.087 & 3.250 & 6.527 & 9.150 & 8.200 & 7.900 & 6.440 & 4.570 & 7.252 \\
\hline Calcium hypochlortie $20 \mathrm{ppm}$ & 9.917 & 8.813 & 6.970 & 5.370 & 3.357 & 6.885 & 10.047 & 8.853 & 7.997 & 6.613 & 4.637 & 7.629 \\
\hline Calcium hypochlortie $40 \mathrm{ppm}$ & 9.040 & 8.030 & 6.310 & 4.300 & 3.043 & 6.145 & 9.070 & 8.147 & 7.240 & 5.707 & 4.420 & 6.917 \\
\hline Calcium hypochlortie $60 \mathrm{ppm}$ & 8.173 & 7.977 & 5.733 & 3.933 & 2.497 & 5.663 & 8.747 & 8.110 & 6.887 & 5.390 & 3.923 & 6.611 \\
\hline Control (Distilled Water) & 7.273 & 6.033 & 4.383 & 2.567 & 1.900 & 4.431 & 6.917 & 6.183 & 6.037 & 4.200 & 3.557 & 5.379 \\
\hline Mean & 9.407 & 8.301 & 6.412 & 5.072 & 3.107 & & 9.235 & 8.279 & 7.612 & 7.336 & 4.369 & \\
\hline Factor & \multicolumn{3}{|c|}{ SEm \pm} & \multicolumn{3}{|c|}{ CD at $5 \%$} & \multicolumn{3}{|c|}{ SEm \pm} & \multicolumn{3}{|c|}{ CD at $5 \%$} \\
\hline Treatments (T) & \multicolumn{3}{|c|}{0.027} & \multicolumn{3}{|c|}{0.078} & \multicolumn{3}{|c|}{0.025} & \multicolumn{3}{|c|}{0.047} \\
\hline Days (D) & \multirow{2}{*}{\multicolumn{3}{|c|}{0.023}} & \multicolumn{3}{|c|}{0.066} & \multicolumn{3}{|c|}{0.021} & \multicolumn{3}{|c|}{0.039} \\
\hline$(\mathrm{T} \times \mathrm{D})$ & & & & \multicolumn{3}{|c|}{0.174} & \multicolumn{3}{|c|}{0.056} & \multicolumn{3}{|c|}{0.104} \\
\hline
\end{tabular}

Table.2 Effect of postharvest application of biocides on water balance and fresh weight change during vase life period of cut gerbera

\begin{tabular}{|c|c|c|c|c|c|c|c|c|c|c|c|c|}
\hline \multirow{3}{*}{ Treatments (T) } & \multicolumn{12}{|c|}{ Days (D) } \\
\hline & \multicolumn{6}{|c|}{ Water Balance (g) } & \multicolumn{6}{|c|}{ Fresh weight change (\% of initial flower weight) } \\
\hline & $2^{\text {nd }}$ & $4^{\text {th }}$ & $6^{\text {th }}$ & $8^{\text {th }}$ & $10^{\text {th }}$ & Mean & $2^{\text {nd }}$ & $4^{\text {th }}$ & $6^{\text {th }}$ & $8^{\text {th }}$ & $10^{\text {th }}$ & Mean \\
\hline Sodium hypochlortie $20 \mathrm{ppm}$ & $\begin{array}{c}4.533 \\
(0.533)\end{array}$ & $\begin{array}{c}4.710 \\
(0.710)\end{array}$ & $\begin{array}{c}3.043 \\
(-0.957)\end{array}$ & $\begin{array}{c}3.243 \\
(-0.757)\end{array}$ & $\begin{array}{c}3.237 \\
(-0.763)\end{array}$ & $\begin{array}{c}3.753 \\
(-0.247)\end{array}$ & 109.710 & 101.613 & 100.100 & 95.447 & 95.447 & 100.463 \\
\hline Sodium hypochlortie $40 \mathrm{ppm}$ & $\begin{array}{c}4.430 \\
(0.430)\end{array}$ & $\begin{array}{c}3.927 \\
(-0.073)\end{array}$ & $\begin{array}{c}3.155 \\
(-0.847)\end{array}$ & $\begin{array}{c}3.040 \\
(-0.960)\end{array}$ & $\begin{array}{c}2.990 \\
(-1.010)\end{array}$ & $\begin{array}{c}3.508 \\
(-0.492)\end{array}$ & 108.433 & 100.930 & 95.220 & 86.253 & 86.253 & 95.418 \\
\hline Sodium hypochlortie $60 \mathrm{ppm}$ & $\begin{array}{c}4.610 \\
(0.610)\end{array}$ & $\begin{array}{c}3.960 \\
(-0.040)\end{array}$ & $\begin{array}{c}2.480 \\
(-1.520)\end{array}$ & $\begin{array}{c}2.647 \\
(-1.353)\end{array}$ & $\begin{array}{c}2.680 \\
(-1.320)\end{array}$ & $\begin{array}{c}3.275 \\
(-0.725)\end{array}$ & 107.943 & 99.807 & 87.833 & 82.687 & 82.687 & 92.191 \\
\hline Calcium hypochlortie 20 ppm & $\begin{array}{c}3.870 \\
(-0.130)\end{array}$ & $\begin{array}{c}3.960 \\
(-0.040)\end{array}$ & $\begin{array}{c}2.973 \\
(-1.027)\end{array}$ & $\begin{array}{c}2.780 \\
(-1.220)\end{array}$ & $\begin{array}{c}2.720 \\
(-1.280)\end{array}$ & $\begin{array}{c}3.261 \\
(-0.739)\end{array}$ & 108.047 & 100.227 & 96.450 & 96.450 & 89.463 & 98.127 \\
\hline Calcium hypochlortie $40 \mathrm{ppm}$ & $\begin{array}{c}3.967 \\
(-0.033)\end{array}$ & $\begin{array}{c}3.920 \\
(-0.080)\end{array}$ & $\begin{array}{c}3.070 \\
(-0.930)\end{array}$ & $\begin{array}{c}2.593 \\
(-1.407)\end{array}$ & $\begin{array}{c}2.623 \\
(-1.377)\end{array}$ & $\begin{array}{c}3.235 \\
(-0.765)\end{array}$ & 107.550 & 98.720 & 95.057 & 95.057 & 85.153 & 96.307 \\
\hline Calcium hypochlortie 60 ppm & $\begin{array}{c}3.427 \\
(-0.573)\end{array}$ & $\begin{array}{c}3.867 \\
(-0.133)\end{array}$ & $\begin{array}{c}2.847 \\
(-1.153)\end{array}$ & $\begin{array}{c}2.547 \\
(-1.453)\end{array}$ & $\begin{array}{c}2.540 \\
(-1.460)\end{array}$ & $\begin{array}{c}3.045 \\
(-\mathbf{0 . 9 5 5})\end{array}$ & 104.223 & 98.353 & 87.047 & 87.047 & 79.327 & 91.199 \\
\hline Control (Distilled Water) & $\begin{array}{c}4.357 \\
(0.357)\end{array}$ & $\begin{array}{c}3.850 \\
(-0.150)\end{array}$ & $\begin{array}{c}2.347 \\
(-1.653)\end{array}$ & $\begin{array}{c}2.367 \\
(-1.633)\end{array}$ & $\begin{array}{c}2.343 \\
(-1.657)\end{array}$ & $\begin{array}{c}3.053 \\
(-0.947)\end{array}$ & 102.050 & 97.727 & 85.170 & 85.170 & 76.073 & 89.238 \\
\hline Mean & $\begin{array}{c}4.170 \\
(0.170)\end{array}$ & $\begin{array}{c}4.028 \\
(0.028)\end{array}$ & $\begin{array}{c}2.845 \\
(-1.155)\end{array}$ & $\begin{array}{c}2.745 \\
(-1.255)\end{array}$ & $\begin{array}{c}2.733 \\
(-1.267)\end{array}$ & & 106.851 & 99.625 & 92.411 & 89.730 & 84.915 & \\
\hline Factor & \multicolumn{3}{|c|}{ SEm \pm} & \multicolumn{3}{|c|}{ CD at $5 \%$} & \multicolumn{3}{|c|}{ SEm \pm} & \multicolumn{3}{|c|}{ CD at $5 \%$} \\
\hline Treatments (T) & \multicolumn{3}{|c|}{0.010} & \multirow{2}{*}{\multicolumn{3}{|c|}{0.027}} & \multirow{2}{*}{\multicolumn{3}{|c|}{$\begin{array}{l}0.011 \\
0.009\end{array}$}} & \multicolumn{3}{|c|}{0.164} \\
\hline Days (D) & \multirow{2}{*}{\multicolumn{3}{|c|}{$\begin{array}{l}0.008 \\
0.021\end{array}$}} & & & & & & & \multirow{2}{*}{\multicolumn{3}{|c|}{$\begin{array}{l}0.138 \\
0.366\end{array}$}} \\
\hline$(\mathrm{T} \times \mathrm{D})$ & & & & \multicolumn{3}{|c|}{0.060} & \multicolumn{3}{|c|}{0.024} & & & \\
\hline
\end{tabular}

Parenthesis represents original values. The data was analyzed statistically after uniform addition of a base value 4.0 
Table.3 Effect of postharvest application of biocides on electrolytes leakage and total sugar content during Vase life period of cut gerbera

\begin{tabular}{|l|}
\hline \\
Treatments (T) \\
\hline Sodium hypochlortie $20 \mathrm{ppm}$ \\
\hline Sodium hypochlortie $40 \mathrm{ppm}$ \\
\hline Sodium hypochlortie $60 \mathrm{ppm}$ \\
\hline Calcium hypochlortie $20 \mathrm{ppm}$ \\
\hline Calcium hypochlortie 40 ppm \\
\hline Calcium hypochlortie 60 ppm \\
\hline Control (Distilled Water) \\
\hline \multicolumn{1}{|c|}{ Mean } \\
\hline Factor \\
\hline Treatments (T) \\
\hline Days (D) \\
\hline (T x D)
\end{tabular}

\begin{tabular}{|c|c|c|c|c|c|c|c|c|c|c|}
\hline \multicolumn{11}{|c|}{ Days (D) } \\
\hline \multicolumn{6}{|c|}{ Electrolytes leakage (\%) } & \multicolumn{5}{|c|}{ Total sugars content (mg/g petal weight) } \\
\hline $2^{\text {nd }}$ & $4^{\text {th }}$ & $6^{\text {th }}$ & $8^{\text {th }}$ & $10^{\text {th }}$ & Mean & $0^{\text {th }}$ & $3^{\text {rd }}$ & $6^{\text {th }}$ & $9^{\text {th }}$ & Mean \\
\hline 18.480 & 24.023 & 27.410 & 33.537 & 35.240 & 27.738 & 2.360 & 4.923 & 5.067 & 2.450 & 3.700 \\
\hline 19.317 & 24.950 & 28.030 & 34.530 & 37.517 & 28.869 & 2.360 & 4.777 & 4.830 & 2.330 & 3.574 \\
\hline 21.320 & 26.163 & 28.240 & 36.760 & 38.050 & 30.107 & 2.360 & 3.547 & 3.723 & 2.080 & 2.928 \\
\hline 20.173 & 25.070 & 29.183 & 35.040 & 37.630 & 29.419 & 2.360 & 3.910 & 3.847 & 2.180 & 3.074 \\
\hline 22.290 & 26.477 & 29.417 & 37.217 & 38.183 & 30.717 & 2.360 & 2.923 & 3.517 & 1.650 & 2.613 \\
\hline 23.300 & 27.350 & 30.150 & 39.183 & 38.977 & 31.792 & 2.360 & 2.793 & 3.327 & 1.447 & 2.482 \\
\hline 24.617 & 28.610 & 30.617 & 39.760 & 39.750 & 32.671 & 2.360 & 2.560 & 2.147 & 1.057 & 2.031 \\
\hline 21.357 & 26.092 & 29.007 & 36.575 & 37.907 & & 2.360 & 3.633 & 3.780 & 1.885 & \\
\hline \multicolumn{3}{|c|}{ SEm $\underline{+}$} & \multicolumn{3}{|c|}{ CD at $5 \%$} & \multicolumn{3}{|c|}{ SEm \pm} & \multicolumn{2}{|c|}{ CD at $5 \%$} \\
\hline \multicolumn{3}{|c|}{0.008} & \multicolumn{3}{|c|}{0.022} & \multicolumn{3}{|c|}{0.006} & \multicolumn{2}{|c|}{0.039} \\
\hline \multicolumn{3}{|c|}{0.007} & \multicolumn{3}{|c|}{0.018} & \multicolumn{3}{|c|}{0.004} & \multicolumn{2}{|c|}{0.029} \\
\hline \multicolumn{3}{|c|}{0.017} & \multicolumn{3}{|c|}{0.049} & \multicolumn{3}{|c|}{0.011} & \multicolumn{2}{|c|}{0.077} \\
\hline
\end{tabular}

Table.4 Effect of postharvest application of biocides on optical density and scape bending during vase life period of cut gerbera

\begin{tabular}{l}
\multicolumn{1}{|c|}{ Treatments (T) } \\
\hline Sodium hypochlortie 20 ppm \\
\hline Sodium hypochlortie 40 ppm \\
\hline Sodium hypochlortie 60 ppm \\
\hline Calcium hypochlortie 20 ppm \\
\hline Calcium hypochlortie 40 ppm \\
\hline Calcium hypochlortie 60 ppm \\
\hline Control (Distilled Water) \\
\hline \multicolumn{1}{|c|}{ Mean } \\
\hline Factor \\
\hline Treatments (T) \\
\hline Days (D) \\
\hline (T x D)
\end{tabular}

\begin{tabular}{|c|c|c|c|c|c|c|c|c|c|c|c|}
\hline \multicolumn{12}{|c|}{ Days (D) } \\
\hline \multicolumn{6}{|c|}{ Optical density } & \multicolumn{6}{|c|}{ Scape bending $\left(^{\circ}\right)$} \\
\hline $2^{\text {nd }}$ & $4^{\text {th }}$ & $6^{\text {th }}$ & $8^{\text {th }}$ & $10^{\text {th }}$ & Mean & $2^{\text {nd }}$ & $4^{\text {th }}$ & $6^{\text {th }}$ & $8^{\text {th }}$ & $10^{\text {th }}$ & Mean \\
\hline 0.014 & 0.017 & 0.031 & 0.042 & 0.065 & 0.034 & 0.000 & 2.243 & 3.850 & 16.870 & 27.120 & 10.017 \\
\hline 0.015 & 0.019 & 0.038 & 0.048 & 0.069 & 0.038 & 0.000 & 4.353 & 10.023 & 18.027 & 30.553 & 12.591 \\
\hline 0.017 & 0.034 & 0.047 & 0.066 & 0.085 & 0.050 & 0.000 & 14.273 & 22.390 & 34.830 & 45.023 & 23.303 \\
\hline 0.014 & 0.024 & 0.044 & 0.056 & 0.098 & 0.047 & 0.000 & 9.343 & 21.730 & 43.390 & 54.587 & 25.810 \\
\hline 0.016 & 0.068 & 0.072 & 0.089 & 0.121 & 0.073 & 0.000 & 17.253 & 24.587 & 49.033 & 62.437 & 30.662 \\
\hline 0.015 & 0.079 & 0.081 & 0.097 & 0.195 & 0.093 & 0.000 & 25.150 & 39.513 & 65.217 & 79.840 & 41.944 \\
\hline 0.016 & 0.085 & 0.093 & 0.105 & 0.221 & 0.104 & 0.000 & 32.083 & 48.027 & 79.350 & 113.97 & 54.687 \\
\hline 0.015 & 0.046 & 0.058 & 0.072 & 0.122 & & 0.000 & 14.957 & 24.303 & 43.817 & 59.076 & \\
\hline \multicolumn{3}{|c|}{ SEm \pm} & \multicolumn{3}{|c|}{ CD at $5 \%$} & \multicolumn{3}{|c|}{ SEm \pm} & \multicolumn{3}{|c|}{ CD at $5 \%$} \\
\hline \multicolumn{3}{|c|}{0.001} & \multicolumn{3}{|c|}{0.002} & \multicolumn{3}{|c|}{$0.02 \overline{8}$} & \multicolumn{3}{|c|}{0.014} \\
\hline \multicolumn{3}{|c|}{0.001} & \multicolumn{3}{|c|}{0.001} & \multicolumn{3}{|c|}{0.023} & \multicolumn{3}{|c|}{0.012} \\
\hline \multicolumn{3}{|c|}{0.001} & \multicolumn{3}{|c|}{0.004} & \multicolumn{3}{|c|}{0.062} & \multicolumn{3}{|c|}{0.031} \\
\hline
\end{tabular}


Table.5 Effect of postharvest application of biocides on vase life of cut gerbera

\begin{tabular}{|l|c|}
\multicolumn{1}{|c|}{ Treatments } & Vase life (days) \\
\hline Sodium hypochlortie $20 \mathrm{ppm}$ & 10.570 \\
\hline Sodium hypochlortie $40 \mathrm{ppm}$ & 7.897 \\
\hline Sodium hypochlortie $60 \mathrm{ppm}$ & 6.490 \\
\hline Calcium hypochlortie $20 \mathrm{ppm}$ & 8.507 \\
\hline Calcium hypochlortie $40 \mathrm{ppm}$ & 7.173 \\
\hline Calcium hypochlortie $60 \mathrm{ppm}$ & 6.270 \\
\hline Control (Distilled Water) & 4.700 \\
\hline Mean & $\mathbf{7 . 3 7 2}$ \\
\hline & \\
\hline SE $\pm m$ & 0.051 \\
\hline CD at 5\% & 0.155 \\
\hline
\end{tabular}

Control recorded significantly highest electrolytes leakage on all the days of observation recorded during vase life period. Improved water balance in the floral tissue reduced stress thus reduced the electrolytes leakage.

Significant changes were observed in the total sugars content of petal tissue of cut gerbera held in different biocide solutions during vase life period (Table 3). Significantly highest total sugars content was recorded in flowers held in sodium hypochlorite at $20 \mathrm{ppm}$ concentration $(3.700 \mathrm{mg} / \mathrm{g}$ petal tissue) followed by sodium hypochlorite $40 \mathrm{ppm}$ ( $3.574 \mathrm{mg} / \mathrm{g}$ petal tissue). Significantly lowest total sugars content in the petal tissue was observed with control treated flowers (2.031 $\mathrm{mg} / \mathrm{g}$ petal tissue). Significant differences were observed in the total sugars content of flower petals during different days of vase life period. Significantly highest total sugars content of flower petals was recorded on day $6(3.780 \mathrm{mg} / \mathrm{g}$ petal tissue) and was found at par with day $3(3.633 \mathrm{mg} / \mathrm{g}$ petal tissue). Significantly lowest total sugars content was recorded on day $9(1.885 \mathrm{mg} / \mathrm{g}$ petal tissue). Interaction effect of total sugars content between days and treatments was also found significant. On day 0 , there were no significant differences for total sugars content among the treatments. Significantly highest total sugars content was observed with sodium hypochlorite at $20 \mathrm{ppm}$ concentration on day 3, 6 and 9 (4.923, 5.067 and 2.450 $\mathrm{mg} / \mathrm{g}$ petal tissue respectively), whereas, significantly lowest total sugars content was observed with control on day 3,6 and 9 $(2.560,2.147$ and $1.057 \mathrm{mg} / \mathrm{g}$ petal tissue respectively). Cut gerbera flowers held in different biocide solutions exhibited certain differences in the biochemical parameters during vase life period. The total sugars content slightly increased from day 0 of experimentation to day 3 and then onwards steadily decreased towards the end of vase life period. The reduced levels of sugars towards the end of vase life period might be due to a decrease in the accumulation of sugars in the floral tissue and reduced translocation into the flower petals. Nichols (1975) also reported similar kind of observation while working with carnation flowers.

Significant differences were observed in the optical density of vase solutions where cut gerbera flowers were held (Table 4). Cut gerbera flowers held in a holding solution containing sodium hypochlorite at $20 \mathrm{ppm}$ concentration recorded significantly lowest 
optical density followed by sodium hypochlorite 40 ppm concentration (0.038). Control recorded significantly highest optical density (0.104). Significant differences were observed in the optical density of vase solutions during different days of vase life period of cut gerbera. On day 2, significantly lowest optical density (0.015) recorded, whereas, on day 10 , significantly highest optical density (0.122) was observed. The interaction effect between days and treatments on optical density of vase solution was also found significant. Cut gerberas held in sodium hypochlorite $20 \mathrm{ppm}$ concentration recorded significantly lowest optical density of vase solution on all the days of observation recorded. Significantly highest optical density of vase solution was however registered with control on all the days of observation recorded except on day day 6. Highest optical density recorded in the control treatment might be due to enormous increase in the microbial growth indicating more turbidity in the vase solution. Humaid (2005) also expressed similar kind of opinion while working with gladiolus flower spikes.

Significant differences were observed in the scape bending curvature of cut gerbera flowers held in different biocide solutions (Table 4). Flowers held in sodium hypochlorite at $20 \mathrm{ppm}$ concentration recorded significantly lowest scape bending curvature (10.017 degrees), whereas, control recorded significantly highest scape bending curvature (54.687 degrees). Significant differences were observed in the scape bending curvature during different days of vase life period of cut gerbera. On day 2, significantly lowest scape bending curvature (0.000 degrees) was observed, whereas, on day 10 , significantly highest scape bending curvature (59.076 degrees) was observed. The interaction effect between days and treatments on scape bending curvature was also found significant. The scape bending curvature was found increased with the passage of the experiment. Sodium hypochlorite at $20 \mathrm{ppm}$ concentration recorded significantly lowest scape bending curvature on almost all the days of observation recorded, followed by sodium hypochlorite $40 \mathrm{ppm}$. Control recorded significantly highest scape bending curvature on almost all the days of observation recorded except on day 2. High turgidity and mechanical strength of the flower scape due to improved water balance in the floral tissue might have led to record lowest scape bending curvature with sodium hypochlorite $20 \mathrm{ppm}$ concentration in the vase solution. Van Meeteran (1979) reported similar kind of observation while working with cut gerberas. Highest scape bending curvature in control treated flowers might be due to poor water relations in the floral tissue due to vascular blockage by microbes thus resulting in lower turgor in the scapes. The present results were found in accordance with the earlier findings of Zieslin et al., (1978) in cut roses, Accati and Jona (1989) and van Doorn et al., (1994) in cut gerbera.

Significant differences were observed in the vase life of cut gerbera flowers held in different biocide solutions (Table 5). Among the treatments, flowers held in sodium hypochlorite $20 \mathrm{ppm}$ recorded significantly longest vase life (10.570 days) followed by calcium hypochlorite $20 \mathrm{ppm}$ concentration (8.507 days). Significantly lowest vase life was however recorded with control (4.700 days). Application of sodium hypochlorite 20 ppm in the vase solution, increased the water uptake, reduced transpiration loss of water, improved the water balance of floral tissue, reduced the electrolytes leakage, improved the sugars content in the floral tissue thus increased the vase life of cut gerbera. Further, effective control of microbial proliferation in the holding solution by addition of sodium hypochlorite at $20 \mathrm{ppm}$ concentration reduced the scape bending curvature thereby improved 
the quality of cut gerbera flowers. The present results were found in accordance with the earlier findings of Babu et al., (2002) who reported an increase in the vase life of Dendrobium flowers due to reduced rate of respiration and prevention of physiological loss in weight.

\section{References}

Accati, E. G. and Jona, R. 1989. Parameters influencing gerbera cut flower longevity. Acta Horticulturae. 261(10): 63-68.

Anju, P. and Santhosh, K. 2004. Response of floral preservatives on postharvest quality of gladiolus spike cultivar "Pink Friendship". Advances in Plant Sciences. 17(2): 529-32.

Babu, D.M., Jawaharlal, M. and Vijayakumar, M. 2002. Influence of holding solutions on the post-harvest life of Dendrobium hybrid Sonia -17. South Indian Horticulture. 50(4/6): 451-57.

Balakrishna, H. V., Venkatesh Reddy, T. and Muthappa Rai, B. G. 1989. Postharvest physiology of cut tuberose as influenced by some metal salts. Mysore Journal of Agricultural Sciences. 23: 344-48.

Bhaskar, V. V. 2001. Effect of chemicals and packing material on the physiological and biochemical changes during flower petal senescence and on vase life of cut rose (Rosa hybrida L.) cv. First Red. Ph. D thesis submitted to Acharya N G Ranga Agricultural University, Hyderabad.

Bhattacharjee, S. K. 1998. Effect of different chemicals in the holding solution on postharvest life and quality of cut roses. Annals of Plant Physiology. 12 (8): 16163.

Buys, C. 1978. Quality problems in gerbera. In: Quagliotti and A Baldi (eds.). Genetics and breeding of carnation and gerbera. Proceedings Eucarpin
Marketing, 24-28 April, Alassio, Italy, pp. 255-62.

Coake, D. L. 1997. Preservatives: A comprehensive guide to fresh flower care and handling products. The American Rose. 34: 15-17.

De Jong, J. 1978. Dry storage and subsequent recovery of cut gerbera flowers as aid in selection for longevity. Scientia Horticulturae. 9: 389-97.

Dubois, M., Gibles, K. A., Hamilton, J. K., Robers, P. A. and Smith, F. 1956. Colorimetric method for determination of sugars and related substances. Analytical Chemistry. 28 (3): 350-56.

Emonger, V. E. 2004. Effects of gibberellic acid on postharvest quality and vase life of cut gerbera flowers (Gerbera jamesonii). Journal of Agronomy. 3(3): 191-95.

Faragher, J. D. 1986. Effects of cold storage methods on vase life and physiology of cut waratah inflorescences (Telopea speciosirsima, Proteaceae). Scientia Horticulturae. 29: 163-71.

Fischer, P., Forchthammer, L. and Kalthoff, F. 1982. Effect of several factors on vase life of cut flowers. Deutscher Gartenbau. 36: 138-41.

Halevy, A. H. and Mayak, S. 1979. Senescence and postharvest physiology of cut flowers-Part 1, pp.204-36. In: J. Janick (ed.) Horticultural Reviews. Vol. I, AVI Publishing, Westport, Conn.

Halevy, A. H. and Mayak, S. 1981. Senescence and postharvest physiology of cut flowers-Part II. Horticultural Reviews. 3: 59-143.

Halevy, A. H., Byrne, T. G., Kofranek, A. M., Farnham, D. S., Thompson, J. F. and Hardenburg, R. C. 1978. Evaluation of postharvest handling methods for transcontinental truck shipments of cut carnations, chrysanthemums, and roses. Journal of American Society for Horticultural Sciences. 103: 151- 56. 
Humaid, M. 2005. Effect of glucose and biocides on vase life and quality of cut gladiolus spikes. Acta Horticulturae. 682(1): 519-25.

Larsen, F. E. and Frolich, M. 1969. The influence of 8-hydroxyquinolene citrate, $\mathrm{N}$-dimethylamino succinamic acid and sucrose on respiration and water flow in 'Red Sim' carnation in relation to flower senescence. Journal of American Society for Horticultural Sciences. 94: 289-91.

Lemper, J. 1981. Postharvest handiling of cut flowers. Land Witskammer Hanuover, German Federal Republic.

Marousky, F. J. 1969. Vascular blockage and moisture retention in cut roses induced by $\mathrm{pH}, 8$-hydroxyquinolene citrate and sucrose. Journal of American Society for Horticultural Sciences. 94:223-26.

Murali, T. P. 1990. Mode of action of metal salts and sucrose in extending the vase life of cut gladioli. Acta Horticulturae. 343: 313-20.

Nichols, R. 1975. Senescence and sugar status of cut flowers. Acta Horticuturae 41:2129.

Panse, V. G. and Sukhatme, P. V. 1985. Statistical methods for agricultural workers. Indian Council of Agricultural Research, New Delhi.

Prashant, P. 2006. Studies on the role of physiological and biochemical components with floral preservatives on the vase life of cut gerbera (Gerbera jamesonii) cv. Yanara. $P h \quad D$ thesis submitted to Acharya N G Ranga
Agricultural University, College of Agriculture, Rajendranagar, Hyderabad. Stoddard, E. M. and Miller, P. M. 1962. Chemical control of water in growing plants. Science. 137: 224-25.

Van Doorn, W. G., Venkat, M. and Bakker, M. L. 1994. Effect of dry storage on scape bending in cut Gerbera jamesonii flowers. Postharvest Biology and Technology. 4: 261-69.

Van Meeteren, U. 1979. Water relations and keeping quality of cut gerbera flowers. Internal water relations of ageing petal tissue. Scientia Horticulturae. 11: 8393.

Wernett, H. C., Sheenan, T. J., Wilfret, G. J., Marousky, P. M., Lyrene, P. M. and Knuft, D. A. 1996. Postharvest longevity of cut flower gerbera. Response to selection for vase life components. Journal of American Society for Horticultural Sciences. 121: 216-21.

Wilberg, B. 1973. Physiological investigation on the problem of freedom from stem break as a pre-requisite in breeding gerbera forms which remain upright when used as cut flowers. Z. Pflanzenzuchung 69: 107-17.

Zieslin, N., Kohl, H. C., Kofranek, A. M. and Halevy, A. H. 1978. Changes in the water status of cut roses and its relationship to bent neck phenomenon. Journal of American Society for Horticultural Sciences. 103: 176-79.

\section{How to cite this article:}

Hema, P., V. Vijaya Bhaskar, A.V.D. Dorajeerao and Suneetha, D.R.S. 2018. Effect of PostHarvest Application of Biocides on Vase Life of Cut Gerbera (Gerbera jamesonii Bolus ex. Hook) cv. Alppraz. Int.J.Curr.Microbiol.App.Sci. 7(03): 2596-2606. doi: https://doi.org/10.20546/ijcmas.2018.703.300 